\title{
Fluorescent biomarkers demonstrate prospects for spreadable vaccines to control disease transmission in wild bats
}

\author{
Kevin M. Bakker ${ }^{1,2 \star}$, Tonie E. Rocke ${ }^{3}$, Jorge E. Osorio ${ }^{4}$, Rachel C. Abbott ${ }^{3}$, Carlos Tello ${ }^{5}$, \\ Jorge E. Carrera ${ }^{6}{ }^{6}$, William Valderrama ${ }^{5,7}$, Carlos Shiva ${ }^{8}$, Nestor Falcon ${ }^{8}$ and Daniel G. Streicker ${ }^{1}{ }^{1,9 \star}$
}

\begin{abstract}
Vaccines that autonomously transfer among individuals have been proposed as a strategy to control infectious diseases within inaccessible wildlife populations. However, rates of vaccine spread and epidemiological efficacy in real-world systems remain elusive. Here, we investigate whether topical vaccines that transfer among individuals through social contacts can control vampire bat rabies-a medically and economically important zoonosis in Latin America. Field experiments in three Peruvian bat colonies, which used fluorescent biomarkers as a proxy for the bat-to-bat transfer and ingestion of an oral vaccine, revealed that vaccine transfer would increase population-level immunity up to 2.6 times beyond the same effort using conventional, nonspreadable vaccines. Mathematical models showed that observed levels of vaccine transfer would reduce the probability, size and duration of rabies outbreaks, even at low but realistically achievable levels of vaccine application. Models further predicted that existing vaccines provide substantial advantages over culling bats-the policy currently implemented in North, Central and South America. Linking field studies with biomarkers to mathematical models can inform how spreadable vaccines may combat pathogens of health and conservation concern before costly investments in vaccine design and testing.
\end{abstract}

\begin{abstract}
nfectious diseases of wildlife cause threats to human and animal health globally ${ }^{1}$. Controlling these pathogens within their natural animal hosts can offer substantial health, economic and conservation benefits. For example, baited vaccines targeting wildlife reservoirs eliminated fox rabies from western Europe ${ }^{2}$ and currently confine raccoon rabies to the eastern United States ${ }^{3}$. However, for many important wildlife diseases, delivery systems to vaccinate a sufficient proportion of host populations to control pathogens are unavailable, and direct (that is, individual-based) vaccination is logistically prohibitive. Interventions that spread from treated to untreated individuals are increasingly used to control arthropod-borne diseases ${ }^{4-6}$ and have been proposed as a solution for the mass vaccination of wildlife, since each unit of vaccine deployed would immunize multiple individuals $s^{7,8}$. However, as was seen with poliovirus eradication efforts, vaccines that sustain transmission may revert to virulent phenotypes ${ }^{9}$, and in wildlife, vaccine shedding may have unanticipated ecological or evolutionary impacts on competing pathogens or host species ${ }^{10}$. Vaccines with deliberately constrained capacity to transmit are therefore currently the preferred candidates for real-world applications. Encouragingly, theoretical models suggest that such weakly transmissible vaccines consistently outperform individual-based vaccination, increasing the potential for disease eradication ${ }^{11}$. Despite this theoretical promise, spreadable vaccines have only rarely been tested in natural systems (namely, rabbit haemorrhagic disease and myxoma virus in rabbits $^{12}$ ). This gap between theory and practice reflects a number of limiting factors: vaccines may be unavailable; epidemiological
\end{abstract}

knowledge of the target pathogen or the dynamics of vaccine spread may be insufficient to guide deployment or predict benefits; and losses incurred under existing management strategies may be considered insufficient to warrant the real or perceived risks of novel interventions.

Vampire bat rabies (VBR)-a universally lethal viral zoonosis found throughout Latin America-represents a tractable system with which to explore the implementation of spreadable vaccines to protect human and animal health. Where common vampire bats (Desmodus rotundus) routinely feed on human blood, VBR is estimated to cause up to 960 deaths per 100,000 people $^{13}$. Losses from livestock mortality exceed US\$50 million annually and disproportionately affect impoverished, rural communities ${ }^{14,15}$. Existing management strategies have been unable to mitigate the burden of VBR. Vaccines for humans and livestock are protective, but high costs and inaccessibility to remote areas limit uptake ${ }^{16}$. Rabies control programmes also cull vampire bats using anticoagulant poisons ('vampiricide'), which are applied in topical gels that spread among bats through social contacts and are ingested during grooming (here, termed 'orotopical transfer') ${ }^{17}$. While culling reduces bat bites on humans and livestock, the effects on rabies transmission remain controversial ${ }^{18,19}$. Moreover, heightened bat dispersal following culls is predicted to exacerbate VBR transmission by increasing the mixing of bat colonies, analogous to the increased transmission of bovine tuberculosis induced through effects of culling on badger home range size $e^{20,21}$. Oral rabies vaccines that spread by the same orotopical mechanism as vampiricide offer an alternative approach.

\footnotetext{
'Institute of Biodiversity, Animal Health and Comparative Medicine, College of Medical, Veterinary and Life Sciences, University of Glasgow, Glasgow, UK. 2Department of Statistics, University of Michigan, Ann Arbor, MI, USA. ${ }^{3}$ US Geological Survey, National Wildlife Health Center, Madison, WI, USA. ${ }^{4}$ Department of Pathobiological Sciences, School of Veterinary Medicine, University of Wisconsin-Madison, Madison, WI, USA. ${ }^{5}$ LLARIY (Asociación para el Desarrollo y Conservación de los Recursos Naturales), Lima, Peru. ${ }^{6}$ Facultad de Ciencias, Universidad Nacional de Piura, Piura, Peru. ${ }^{7}$ Universidad Autonoma de Barcelona, Barcelona, Spain. ${ }^{8}$ Facultad de Medicina Veterinaria y Zootecnia, Universidad Peruana Cayetano, Lima, Peru. ${ }^{9}$ MRC-University of Glasgow Centre for Virus Research, Glasgow, UK. ^e-mail: bakkerke@umich.edu; daniel.streicker@glasgow.ac.uk
} 

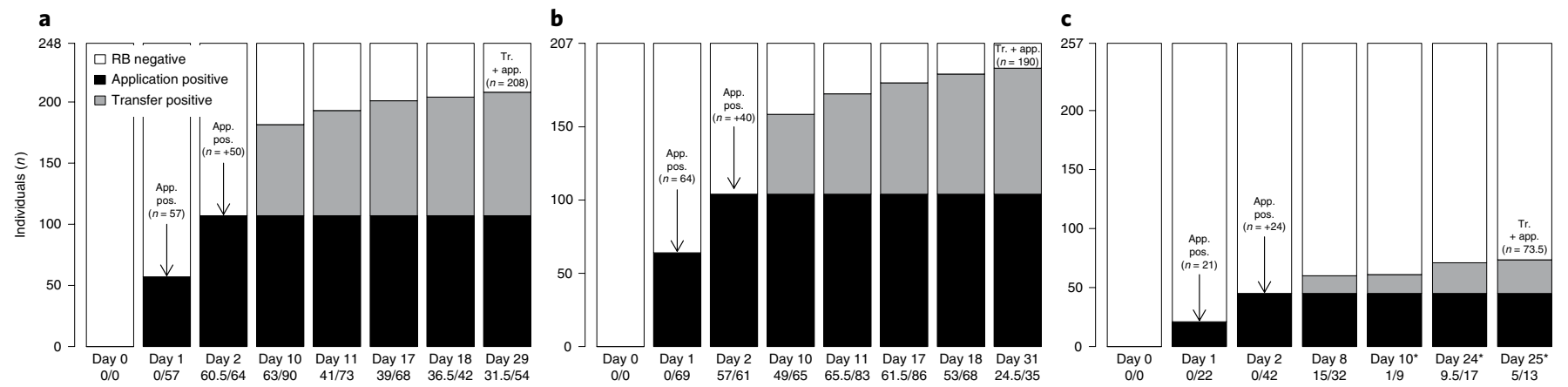

Fig. 1 | Transfer and ingestion of an orotopically spread gel biomarker in three vampire bat colonies. a-c, Transfer and ingestion data for the gel biomarker are shown for LMA5 (a), LMA6 (b) and LMA12 (c). Days on the $x$ axes show the time since RB application, with the number of transfer-positive bats over total captures marked underneath. The yaxes show the numbers of bats in each colony within three categories: RB negative (white); application positive (black); and transfer positive (grey). Asterisks on and after day 10 in c indicate captures from the relocated roost. Data are the means of microscopy readings from two observers, except where noted otherwise. Transfer-positive bats from day 2 had RB applied and were included in the black bar to visualize the total force of application, but were included as transfers in the statistical analyses.

These recombinant virally vectored vaccines can indirectly immunize untreated bats in captivity, but have never been tested in wild populations ${ }^{22-25}$. Several unresolved questions must be answered before deploying vaccines for large-scale bat rabies control: (1) How efficiently would vaccines transfer among wild bats?; (2) Are certain demographic groups of bats especially difficult to vaccinate or especially effective disseminators of vaccines?; (3) Would the resulting degree of immunization significantly reduce rabies transmission?; and (4) Would vaccines reduce human and livestock rabies risk more effectively than the current policy of culling? We address these questions by coupling field studies that used fluorescent biomarkers to quantify contact networks and orotopical transfer among wild vampire bats with mathematical models that simulated how vaccines and vampiricide, which spread by identical mechanisms, would impact the size, duration and probability of rabies outbreaks.

\section{Results}

Biomarker transfer and ingestion show potential for high vaccine coverage in wild vampire bats. We estimated the potential for a spreadable vaccine to transfer among bats using rhodamine $b$ (RB) - a biomarker that, when ingested, leads to long-lasting fluorescence in hair follicles in diverse mammalian species ${ }^{26-28}$. After applying a gel formulation of RB topically to bats in three colonies in Peru (colony sizes: 207-257 individuals; sex ratios: $43.1-50.6 \%$ male), orotopical transfer and ingestion were monitored by fluorescent microscopic analysis of hair samples collected in subsequent capture sessions, with fluorescence indicating RB consumption (Supplementary Table 1). At two sites (LMA5 and LMA6), an estimated 84 and $92 \%$ of bats, respectively, ingested RB, either following topical application or transfer from treated bats (Fig. 1). The third colony (LMA12) relocated to an undocumented roost soon after RB treatment, which diminished captures during the monitoring period relative to the estimated colony size (Supplementary Table 1). Consequently, the overall estimated coverage dropped to $28.8 \%$ (Fig. 1). Nevertheless, the percentage of sampled LMA12 bats at the end of the 1-month monitoring period that were RB positive (48.3\%; aggregating days 24 and 25 ) was not statistically different from the percentages at the final capture dates in the other two colonies (58.3 and 70.0\%; chi-squared test, $\chi^{2}=3.2$; d.f. $=2$; $P=0.21)$. We further characterized patterns of RB uptake among demographic groups of bats. The sex ratios of transfer-positive bats became slightly more male biased (3-11\% increases, depending on the colony) relative to the sex ratios of bats that were treated with $\mathrm{RB}$, suggesting elevated transfer to males; however, these increases were not statistically significant $\left(\chi^{2}\right.$ tests; all $P>0.05$; Supplementary
Fig. 1). We observed RB transfer to untreated bats in all three age classes. Across all colonies, $73.4 \%$ of sampled adults $(\bar{n}=351$, averaged across microscopy readings of independent observers), $57.5 \%$ of sampled juveniles $(\bar{n}=30.5)$ and $89.9 \%$ of sampled subadults $(\bar{n}=34.5)$ became RB positive through transfer during the monitoring period. Consequently, these results implied that vaccines deployed over only $2 \mathrm{~d}$ of captures (17-50\% of the total colony size) would yield high levels of population immunity across age classes due to orotopical transfer.

Contact heterogeneities among demographic groups of vampire bats. Next, we examined whether contact heterogeneities might make certain demographic groups of bats especially effective or ineffective spreaders of vaccines using ultraviolet powder marking, wherein different age and sex groups of bats were treated with different colours of ultraviolet powder, and transfer to untreated bats was monitored over two subsequent capture nights ${ }^{29,30}$. Across three replicate ultraviolet treatments per colony, we documented 78 instances of ultraviolet powder transfer, leading to estimated contact rates ranging from $0.23-1.25$ per treated bat (Fig. 2). Male bats had significantly higher contact rates than female bats (Wilcoxon ranksum test, $W=91 ; P=0.025$; mean $=1.14$ versus 0.67$)$ and had similar rates of male-to-male and male-to-female contacts (Wilcoxon rank-sum test, $W=42 ; P=0.93)$. In contrast, females preferentially contacted other females (Fig. 2a). Transfer to juveniles could not be reliably quantified because these bats were mostly too young to forage independently, and our capture method during the monitoring period required bats to fly out of their roosts. Nevertheless, a single captured juvenile bat had ultraviolet transfer from a female. In contrast, transfer from juveniles to adults should have been detectable if it occurred due to the greater ease of capturing adults. However, none of the 27 marked juveniles transferred ultraviolet powder to adults. Together with the high observed rates of juvenile exposure to $\mathrm{RB}$, these findings suggest that vaccine deployments should target adults rather than juveniles. Targeting adults would further be logistically advantageous since it would minimize the social disruption of colonies that results from entering roosts to capture juveniles.

Epidemiological models show that spreadable vaccines outperform culling for rabies control. We adapted a deterministic compartmental model of VBR persistence ${ }^{20}$ to incorporate an orotopically spread vaccine, and used least squares (Fig. 3b) to estimate the expected per-capita vaccine transfer rates from the time series of RB transfers observed in our field studies, assuming that RB transfer equated to lifelong protection. This analysis revealed that each 
a

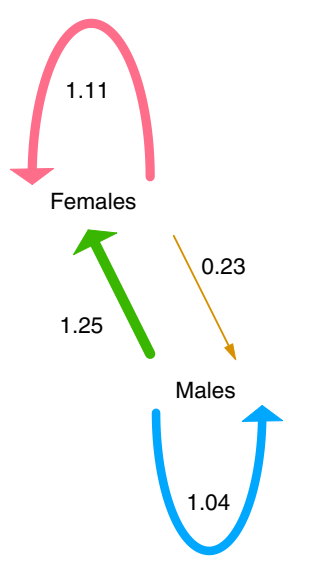

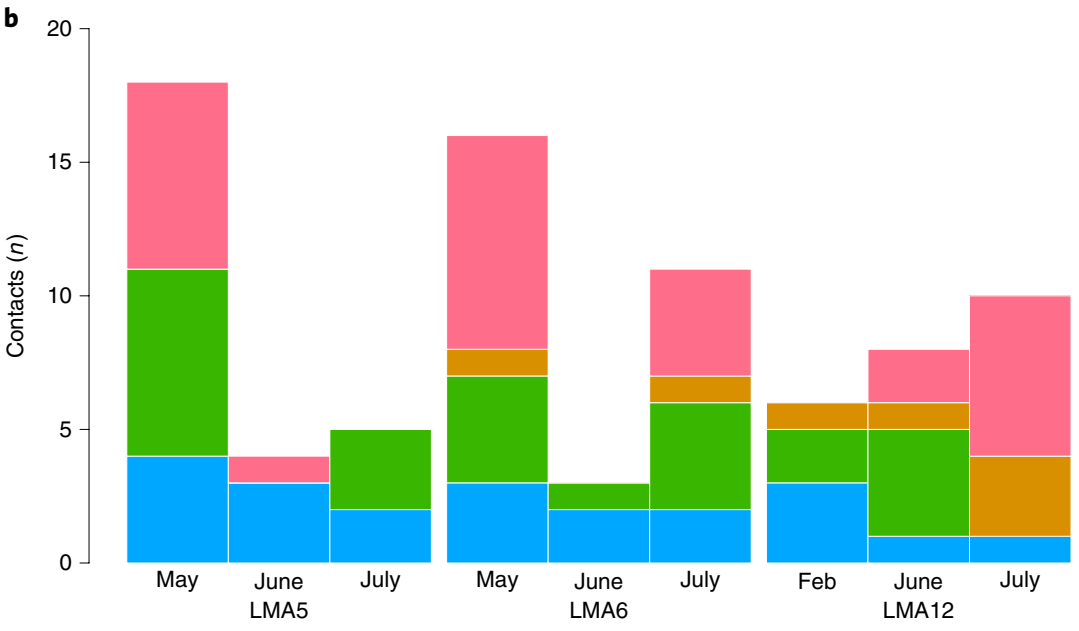

Fig. 2 | Bat contact heterogeneity revealed by ultraviolet powder transfers. a, Mean new contacts per marked bat, by sex. Arrow thicknesses are proportional to contact rates. $\mathbf{b}$, Number and directionality of contacts by sex, location and sampling date. Contacts to juveniles are not shown since the juveniles in the studied colonies were too young to feed independently and would have been underestimated by our capture method during monitoring.

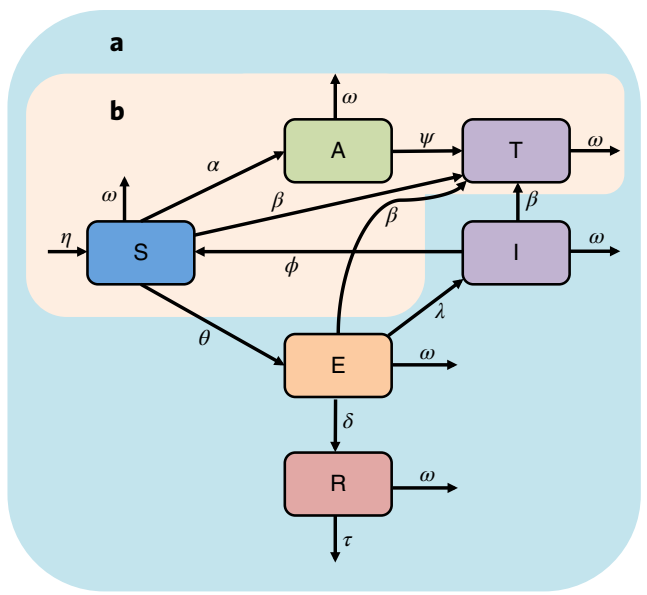

Fig. 3 | Dynamic models of rabies transmission and spreadable vaccination. a, The full model used for the outbreak analyses included orotopical transfer and rabies transmission. Classes comprised susceptible $(S)$, application-positive (A), transfer-positive ( $T$ ), immune (I), exposed to rabies $(E)$ and rabid bats $(R)$. b. Biomarker transfer model structure for fitting $\beta$. In the vaccination model, the I and T classes both provide immunity from rabies, but the T class has permanent immunity. Model parameters describe the rates of: natural births $(\eta)$ and deaths $(\omega)$; orotopical gel application $(\alpha)$, persistence $(\psi)$ and transfer $(\beta)$; rabies transmission $(\theta)$; waning of immunity $(\phi)$; rabies-induced mortality $(\tau)$; and the probabilities of succumbing to rabies $(\delta)$ or surviving $(\lambda)$ following exposure. Supplementary Table 3 provides further details and references for parameter values.

treated bat transferred RB to 1.45-2.11 untreated individuals-up to a 2.6-fold increase in population-level coverage relative to the coverage that would be expected using conventional, non-spreading vaccines (Fig. 4b, Supplementary Fig. 2 and Supplementary Table 2). We simulated the ability of spreadable vaccines to control rabies across the range of $R_{0}$ (the expected number of secondary infections arising from a single viral introduction into a completely susceptible population) values (0.6-2.0) suggested in the rabies literature ${ }^{20,31,32}$. Applying vaccines to approximately $20 \%$ of bats vaccinated $40 \%$ of the population and reduced rabies outbreak size by $45-75 \%$, depending on the assumed $R_{0}$ of rabies (Fig. $4 \mathrm{a}-\mathrm{c}$ ). However, applying vaccines to a higher proportion of bats had diminishing returns for both the proportion of the colony that was ultimately protected and for rabies control. If vaccines were applied to $>30 \%$ of bats, additional reductions in rabies outbreak sizes were $<5 \%$, meaning that a $5 \%$ increase in initial application led to a $<5 \%$ reduction in outbreak sizes (Fig. 4d). The greatest benefit (reduction in outbreak size relative to effort) occurred at vaccination levels $<15 \%$.

Next, we compared the relative efficacy of vaccination and culling across three epidemiological scenarios representing different management strategies: (1) a preventative approach, where vaccine/ vampiricide was applied to prevent VBR invasion into historically rabies-free bat populations ${ }^{33,34}$; (2) a proactive approach, which represented an intervention in a VBR endemic area, but in a colony that was not currently infected; and (3) a reactive approach where intervention followed $60 \mathrm{~d}$ after a single VBR-infected bat was introduced to the colony (Supplementary Fig. 4). Although we simulated outcomes across the full possible range of application efforts (that is, $0-100 \%$ of bats treated), we focused on lower application levels since capturing large proportions of bats across large geographic areas would be impractical for rabies control campaigns. Indeed, markrecapture studies across multiple vampire bat colonies in Peru suggested that, on average, $<10 \%$ of colonies were captured in a single night $^{19}$. At realistic levels of application, vaccination consistently reduced the probability of viral invasion, outbreak size and outbreak duration more effectively than culling, regardless of whether control measures were preventative, proactive or reactive (Fig. 5). Culling was only favoured when at least $25 \%$ of the colony was treated, and only in reactive scenarios. However, the advantage of culling on outbreak size was relatively small (a maximum of a $20 \%$ greater reduction) relative to the larger advantages observed when vaccination was favoured (up to $45 \%$ greater reduction), and differences in outbreak duration were negligible until much larger proportions of bats were culled (Fig. 5). For the preventative and proactive scenarios, culling required the capture and treatment of much larger proportions of vampire bat populations (for example, $>60 \%$ ) to match the reduction in outbreak size and duration achieved by vaccination (Fig. 5). In fact, the only discernible difference at higher application levels was a greater reduction in the duration of outbreaks by culling; however, this was due to near-complete extinction of bat colonies. Even if this degree of bat culling were achievable and ethically acceptable, it may not be a favourable long-term strategy since 

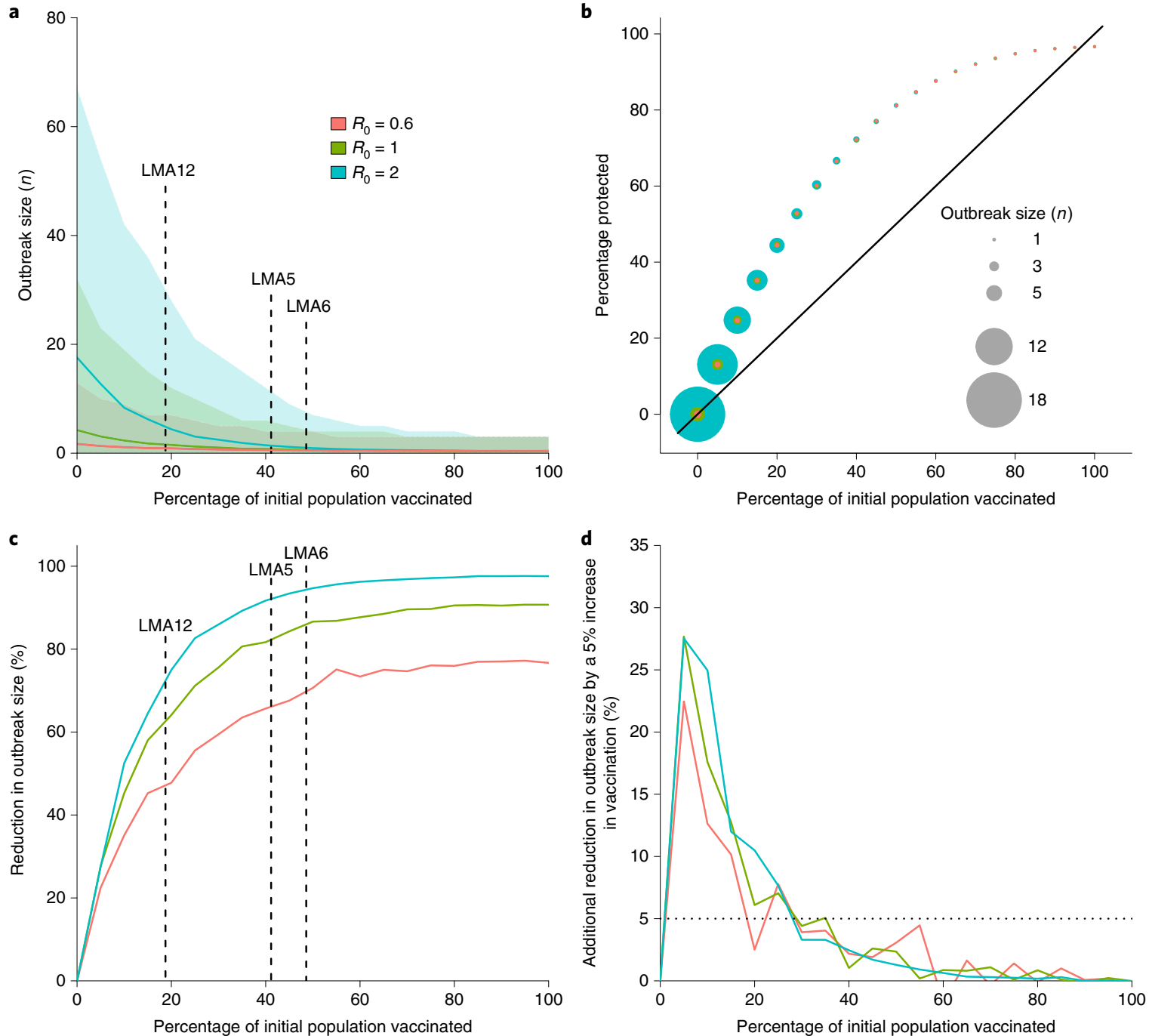

Fig. 4 | Simulating rabies outbreaks with vaccination. a, Mean rabies outbreak sizes after the introduction of a single rabid bat to the colony 1 week following the application of a spreadable vaccine. Colours represent varying degrees of rabies $R_{0}$, with $95 \%$ confidence intervals (shading) calculated from 5,000 simulations. Dashed lines indicate the percentage of bats that RB was applied to at our study sites. Supplementary Fig. 5 shows the results calculated only from simulations where outbreaks occurred. b. Percentage of bats ultimately protected by initial vaccine application. Circle sizes indicate outbreak sizes for the three rabies $R_{0}$ values. The solid line represents $x=y$. Points above the line represent the added benefit of vaccine transfer. c, Reduction in rabies outbreak size (\% fewer cases) for varying initial vaccination levels and rabies $R_{0}$ values. d, Percentage of additional rabies cases prevented by increasing the initial vaccine application effort by $5 \%$ (that is, the rate of change in rabies reduction from $\mathbf{c}$ ).

populations that recovered from culls would be entirely susceptible to rabies, potentially causing larger future outbreaks ${ }^{35}$.

Our per-capita transfer rates probably represent lower bounds of vaccine and vampiricide spread, since the relatively high percentage of bats initially treated with RB left few others available to be exposed via transfer in two of our colonies, and relocation of the third colony reduced capture rates during the monitoring period. Indeed, some studies have suggested higher transfer rates of vampiricide ${ }^{17,36}$. Therefore, we conducted a sensitivity analysis in which both vaccines and vampiricide spread up to tenfold more efficiently than in our RB estimates (values that exceeded the largest transfer rates suggested from vampiricide applications). Additionally, we considered transfer rates that were up to $75 \%$ less efficient than our RB estimates. This analysis showed that low-level vaccination remained favoured under preventative and proactive approaches even if both the vaccine and vampiricide spread up to threefold greater than was observed in our field studies (Supplementary Figs. 6-8). If both interventions spread less effectively than RB, vaccination was either superior or equivalent to culling, except when large proportions of bat colonies were reactively culled (Supplementary Fig. 6). Under realistic levels of application (application $\leq 25 \%$ ), even if vampiricide spread threefold better than a vaccine, it was unable to outperform vaccination under preventative or proactive approaches when $R_{0}$ was $<2$. Under reactive scenarios, culling was favoured if vampiricide spread two- to threefold better than a vaccine or if the VBR $R_{0}$ was 2 (Supplementary Fig. 9). Given that existing oral rabies vaccines use replication-competent viral vectors with the potential for lower effective doses than chemical poisons $s^{24,25}$, heightened vampiricide transfer is less likely than the converse where vaccines spread better ${ }^{8}$. The high $R_{0}$ scenarios where culling is favoured are also unlikely, as the estimated VBR $R_{0}$ is considerably lower than 2 (ref. ${ }^{20}$ ). Our results therefore support previous suggestions that culling may require near-elimination of bats to locally benefit rabies prevention ${ }^{18}$, and reveal spreadable vaccines as efficient tools to reduce the size, duration and probability of rabies outbreaks in Latin America. 
a

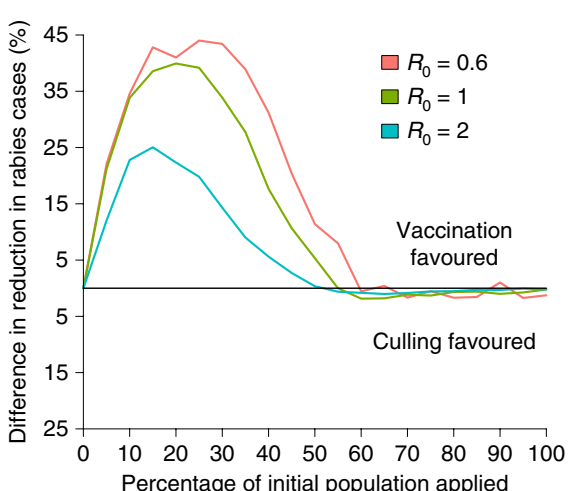

d
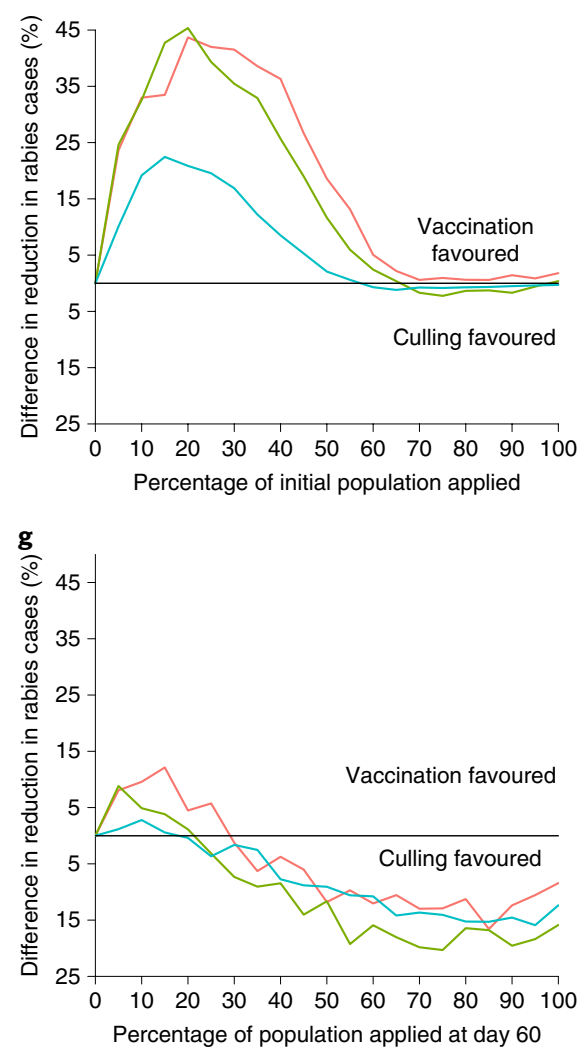

b

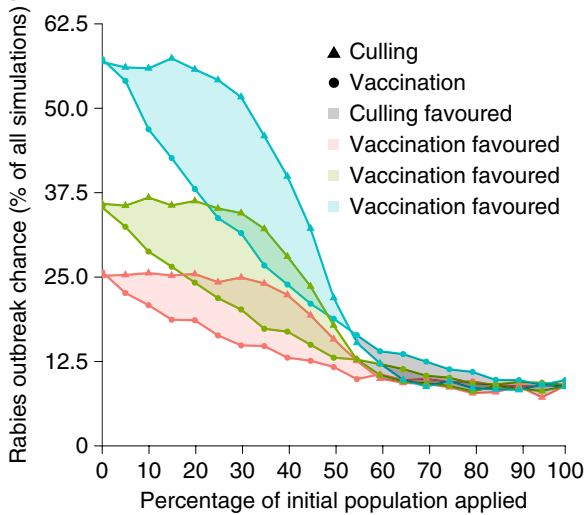

e

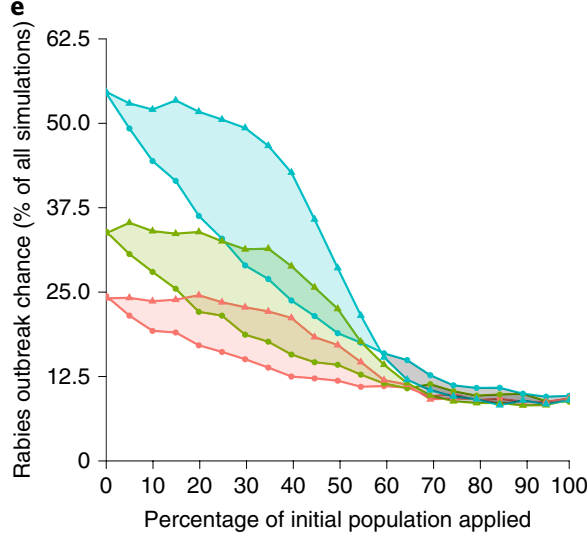

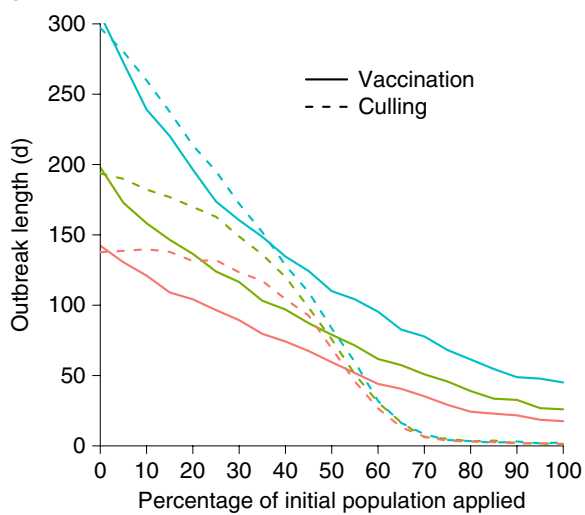

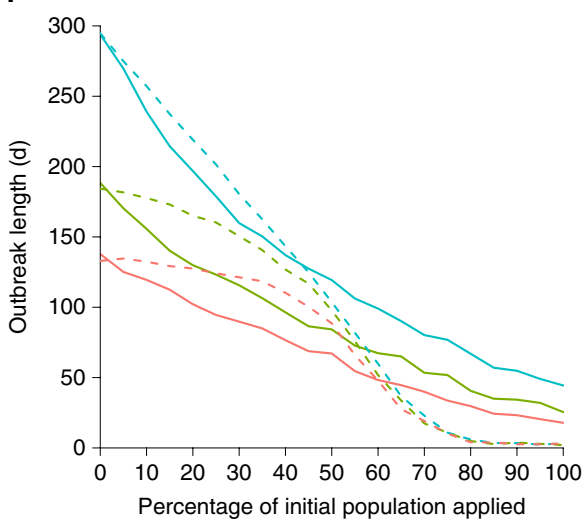

h

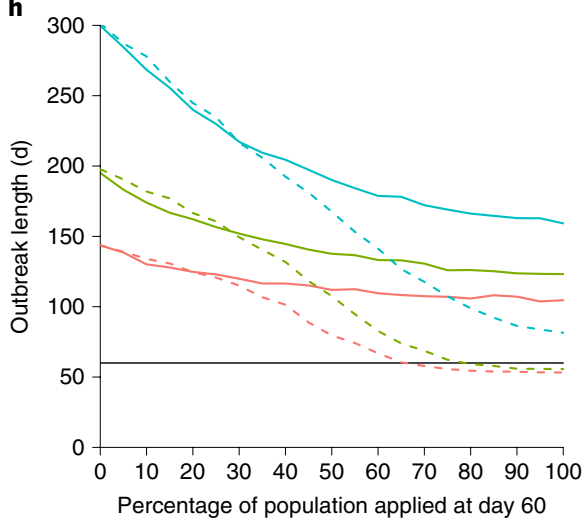

Fig. 5 | Comparison of the effects of culling and vaccination on rabies transmission. a-h, Difference in the reduction of rabies cases between equal levels of effort in vaccination versus culling ( $\mathbf{a}, \mathbf{d}$ and $\mathbf{g}$ ), probability of a rabies outbreak ( $\mathbf{b}$ and $\mathbf{e}$ ) and duration of rabies outbreaks following vaccination and culling (c, $\mathbf{f}$ and $\mathbf{h}$ ) for the preventative (a-c), proactive ( $\mathbf{d}-\mathbf{f})$ and reactive ( $\mathbf{g}$ and $\mathbf{h}$ ) strategies. In $\mathbf{a}, \mathbf{d}$ and $\mathbf{g}$, values above and below 0 favour vaccination and culling, respectively. In $\mathbf{b}$ and $\mathbf{e}$, the probability of a rabies outbreak is defined as the percentage of simulations ( $n=5,000$ ) where VBR virus introduction led to onward transmission, and shaded regions represent the difference between vaccination (circles) and culling (triangles); culling is favoured in the grey regions and vaccination is favoured in the blue, green and red regions. The probability of outbreaks was not modelled for reactive control since, by definition, outbreaks had already occurred. The horizontal line in $\mathbf{h}$ indicates day 60, when reactive control measures were implemented. In all panels, colours correspond to different assumed $R_{0}$ values for rabies.

\section{Discussion}

This study provides proof of principle that at operationally achievable levels of deployment and empirically quantified rates of batto-bat spread, orotopical vaccines should reduce rabies transmission more effectively than culling (the current policy employed across Latin America). Since VBR persistence requires inter-colony spread for viral dispersal, even modest reductions in outbreak size are likely to have epidemiologically important impacts at the larger geographic scales over which disease control campaigns are implemented. In particular, by reducing the number of infected bats and the probability of viral invasion, vaccination of a limited number of colonies would disproportionately benefit regional rabies elimination by favouring stochastic viral extinctions. Because male dispersal spreads rabies between colonies, vaccination might further benefit from targeting male bats ${ }^{33}$. Although higher rates of social grooming among females were expected to undermine this strategy $y^{36,37}$, we found that males have equal or greater inter- and intra-sex contact rates-a possible consequence of attempted mating 
with females or fighting among males. Importantly, because selfgrooming is common $^{38}$, any vaccine transferred through these interactions would ultimately be ingested.

Designing large-scale campaigns to deploy spreadable rabies vaccines requires additional research in several areas. First, to optimize the number of vaccine doses to apply to each bat, captive and field studies should quantify individual heterogeneity in transfer rates using actual vaccines in addition to biomarkers. Second, the costs of vaccination must be estimated in economic terms in addition to the epidemiological assessment provided here. Unfortunately, vaccines are currently produced only for research, and the costs of large-scale production are unavailable. Third, vaccination of vampire bats without population reduction will be unacceptable to some stakeholders since uncontrolled bat depredation sustains exposures to non-rabies pathogens ${ }^{39}$, and anaemia from bites may reduce livestock productivity independent of rabies ${ }^{40}$. Given that culling shifts bat populations towards younger, more rabies-susceptible individuals, which could enhance rabies transmission ${ }^{19}$, future research should develop tools for reproductive suppression as an alternative to culling ${ }^{41}$. Finally, metapopulation maintenance of rabies provides opportunities for more efficient, epidemiologically informed vaccination ${ }^{42}$. For example, vaccines might be deployed with previous knowledge of rabies presence from livestock surveillance systems (for example, ring vaccination) or preventatively in areas where the locations and timings of outbreaks are predictable ${ }^{34}$. Spatially explicit rabies transmission models will be an important next step in the design of these interventions, but will require a more quantitative understanding of bat dispersal than is currently available. Excitingly, once strategies are developed, the operational capacity for their implementation is already available in most Latin American countries as the result of decades of experience with culling campaigns.

These results provide evidence that spreadable vaccines may contribute to pathogen management within wild bats. VBR provided an ideal case study because the epidemiological mechanisms underlying viral maintenance are understood and candidate vaccines are available $e^{20,25,34,43}$. While the exact parameter estimates and models developed here should not be applied directly to other bat pathogens, the framework linking biomarkers to mathematical models can guide future research. For several bat pathogens of public health or conservation concern, such as white nose syndrome, Hendra virus and Marburg virus, epidemiological models have been proposed ${ }^{4-46}$, and vaccines for bats either exist or have precedents encouraging their development ${ }^{47-49}$. In these cases, our approach could be implemented over relatively short timescales to evaluate the prospects for vaccines to aid management and the immunological and epidemiological characteristics that would be required for success before investing resources in vaccine development. For other bat pathogens with greater uncertainty in reservoir hosts and transmission biology, such as ebolaviruse ${ }^{50}$, implementation will require greater fundamental knowledge of viral transmission cycles. We encourage further development of virally vectored vaccines for bats, and highlight the need to quantify their spread and efficacy in the wild.

\section{Methods}

Field studies of biomarker transfer and ingestion. Field studies were carried out between January and July 2017 in three vampire bat roosts in the Barranca (LMA5 $10^{\circ} 38^{\prime} 29.4^{\prime \prime} \mathrm{S}, 77^{\circ} 48^{\prime} 57.6^{\prime \prime} \mathrm{W}$ ), Huaura (LMA6: $11^{\circ} 03^{\prime} 19.8^{\prime \prime} \mathrm{S}, 77^{\circ} 27^{\prime} 33.8^{\prime \prime} \mathrm{W}$ ) and Lima (LMA12: $12^{\circ} 10^{\prime} 59.9^{\prime \prime} \mathrm{S} 76^{\circ} 50^{\prime} 60^{\prime \prime} \mathrm{W}$ ) provinces of the Department of Lima, Peru (Supplementary Table 1). Two roosts (LMA5 and LMA6) had been monitored since 2007, while the third (LMA12) was examined here for the first time ${ }^{19}$. All roosts were manmade tunnels that formed part of crop irrigation systems. Diurnal captures were carried out to mark the bats and estimate sex ratios and colony sizes. Diurnal captures involved teams entering caves and catching bats with hand nets (BioQuip; Tropics Net). In addition, 2.5-m mist nets (Ecotone) were placed at each end of the tunnels to catch the bats that attempted to escape. Diurnal capture efforts were set to $1 \mathrm{~h}$ across sampling dates and localities. Colony sizes were estimated using the Schnabel method ${ }^{51}$. Nocturnal captures were carried out in the same roosts to monitor biomarker spread. Nets placed at each roost exit were checked every $30 \mathrm{~min}$ for $4 \mathrm{~h}$ per night at varying hours depending on the lunar cycle. Following removal from mist nets, bats were placed in individual cloth bags until processing. All captured bats were given an individually numbered, four-digit Incoloy wing band ( $3.5 \mathrm{~mm}$; Porzana) to identify recaptures. Age was classified using the terms juvenile, subadult or adult, based on the degree of fusion of the phylangeal epiphyses ${ }^{52}$. In total, we recorded 1,777 captures of 709 individually marked bats, with the average bat captured 2.39 times (range $=1-9$ ).

Studies of vaccine transfer and ingestion used RB powder $(50 \mathrm{mg})$ mixed with glycerine jelly ( $44.5 \mathrm{ml}$; Carolina Biological Supply Company) and water $(55.5 \mathrm{ml})$ to form a gel. On days 1 and 2, RB was administered orally to confirm fluorescence in $\mathrm{RB}$-treated bats $(\sim 0.05 \mathrm{ml}$ via needle-free syringe) and applied topically (around $0.45 \mathrm{ml}$, rubbed into the dorsal fur) to all captured bats. Uptake in untreated bats was monitored using hair plucked from bats captured over 4-5 subsequent sessions per colony, carried out up to $31 \mathrm{~d}$ after initial application (Supplementary Table 1). Hair samples were examined with a Nikon SMZ1270 microscope at $15 \times$ using a fluorescence filter with an excitation wavelength of $540 \mathrm{~nm}$ and an emission wavelength of $625 \mathrm{~nm}$. Each sample was examined by two individuals to minimize misclassification, except at LMA12 on days 8 and 10, when only one individual examined the hair. The presence of fluorescence in hair was interpreted to indicate the transfer and consumption of RB, but was not considered a quantitative measure of the volume of RB consumption. Because bats had identification tags, we were able to distinguish those that were positive due to transfer from RB-treated bats (that is, transfer positives) from those that had RB applied by experimenters (that is, application positives). Hair samples were collected under the Peruvian collection permit 028-2017-SERFOR/DGGSPFFS and exported to the United States under export permit 3235-SERFOR. This research was performed with the approval of the University of Glasgow School of Veterinary Medicine Animal Ethics Committee (project 25A/18).

Contact heterogeneities among demographic groups of vampire bats. Powder marking was replicated three times per colony (for a total of nine marking sessions) and bats were monitored for two nights following each marking session (Supplementary Table 1). During each session, red, green, blue or orange ultraviolet powder (DayGlo) was rubbed into the fur of the bat across the entire body using a toothbrush, with colours dependent on age and sex. Ultraviolet colours were rotated between groups for different capture dates to control for potential differences in detection probability. Ultraviolet powder markings were recorded by examining each captured bat for $30 \mathrm{~s}$ using handheld ultraviolet lights (Glowtech) before removal from mist nets. After removing ultraviolet-marked bats from the recaptures, directional contact rates for each sex (for example, female-tomale contacts per marked female) were calculated using equation (1):

$$
\text { Contact rate }=\frac{\frac{n_{\mathrm{pos} X}}{\mathrm{UM}_{X}} * n_{\mathrm{UM}}}{M_{X}}
$$

where $n_{\text {pos }_{x}}$ is the number of bats of a certain sex testing positive for the ultraviolet colour in question, $\mathrm{UM}_{X}$ is the number of unmarked bats of that sex captured at this time point, $n_{\mathrm{UM}_{X}}$ is the number of unmarked bats of that sex in the entire colony, and $M_{X}$ is the number of initially marked bats from that sex. Example calculations are provided in the Supplementary Information (equations (2) and (3)).

Sex biases in ultraviolet transfer were tested by comparing all estimated rates from males with all estimated rates from females, treating each site, month and recipient sex combination as independent observations $(n=36)$. We used a nonparametric Wilcoxon rank-sum test since rates were not normally distributed, even after $\log$ transformation (Shapiro-Wilk test, $P=0.01$ ).

Parameter estimation and mathematical modelling. Per-capita rates of orotopical transfer and ingestion, defined as the estimated number of bat-to-bat transfers per treated individual, were estimated using the data from our RB field study. Specifically, we incorporated a susceptible (S), application-positive (A) and transfer-positive (T) deterministic compartmental model (Fig. 3b) using leastsquares methods in the statistical software R. A 2-d transfer period was integrated with the number of RB application and transfer positives across time to estimate the expected transfer rate of orotopical vaccines or poisons $(\beta)$. A 6-d RB transfer period was also considered to examine variation in $\beta$ across time (Supplementary Table 2 and Supplementary Information). We assumed that successful transfer led to death in the culling models and lifelong protection against VBR in the vaccination models ( 3.5 years of protection given the lifespan of D. rotundus; Supplementary Table 3). Importantly, waning of vaccine-induced immunity would not alter the results shown here, which focused on single outbreaks.

Mathematical models of rabies control used a stochastic model that simulated both rabies transmission and vaccine transfer. A susceptible (S), applicationpositive (A), transfer-positive (T), exposed to rabies (E), immune (I) and rabid (R) model, with a daily time step, was simulated for 5,000 iterations using a Gillespie algorithm (Fig. 3a and Supplementary Information). Following previous models of $\mathrm{VBR}^{20}$, and consistent with the absence of strong relationships between colony size and rabies seroprevalence ${ }^{19}$, we utilized a frequency-dependent rabies transmission 
function. We used 237 bats as the colony size (the mean size from our three field sites). The base model without vaccination or culling followed the mathematical structure and parameter values used by Blackwood et al. ${ }^{20}$, with the simplifications of a single infectious class and modelling of a single introduction of rabies rather than sustained introductions via immigration. This model generated similar outbreak dynamics to the Blackwood et al. ${ }^{20}$ model, characterized by short-lived outbreaks ( $<1$ year) followed by viral extinction, persistence of the bat population, and seroprevalence levels consistent with field observations, particularly at values of $R_{0}>0.6$ (Supplementary Fig. 3). Since we modelled our vaccine spread on a recombinant raccoonpox virus-vectored vaccine that appears unlikely to spread via an infectious process (that is, from indirectly vaccinated bats) ${ }^{43}$, vaccines were modelled to spread only from those bats to which the vaccine was applied, creating a single generation of transmission. Based on the very low prevalence of rabies in free-flying bats $(<1 \%)$ and infrequent dispersal in vampire bats ${ }^{53,54}$, we simulated the introduction of a single rabid bat to the population. Given that sex differences in RB transfer were non-significant, and age-biased transfer was difficult to quantify due to small sample sizes in non-adult classes, we opted against more complex age- and sex-structured models of rabies and vaccine/vampiricide spread.

Models comparing the efficacies of vampiricide and vaccination used the same model structure with the exception that bats in the exposed class died from ingesting vampiricide, while those that consumed the vaccine were not protected (see equations (8) and (9) in the Supplementary Information). This was because post-exposure vaccination has not been evaluated in bats. We generally assumed equal transfer rates of vaccines and vampiricide based on their identical mechanism of transfer; however, we relaxed this assumption in the Supplementary Information (Supplementary Fig. 9). We also assumed that both spread over relatively short time periods, since vampire bats are exceptional groomers and would quickly ingest vaccine or vampiricide ${ }^{38}$. Importantly, our focal vaccine remains viable over these timescales ${ }^{55}$. After 2 years $(730 \mathrm{~d})$, the cumulative number of newly infected bats was considered to be the outbreak size. Outbreak duration was defined as the total number of days when at least one bat was in the exposed class. For preventative and proactive approaches, we quantified the probability of an outbreak as the proportion of simulations in which at least one new bat became infected after a single rabid bat was introduced.

Reporting Summary. Further information on research design is available in the Nature Research Reporting Summary linked to this article.

\section{Data availability}

Source data for Figs. 1 and 2 are provided with this Article at https://doi. org/10.1038/s41559-019-1032-x. The complete dataset describing ultraviolet and RB transfer is available from Dryad (https://doi.org/10.5061/dryad.64t161m).

\section{Code availability}

The R scripts used to estimate the RB transfer rates shown in Supplementary Fig. 2 and Supplementary Table 2 and to carry out the epidemiological modelling shown in Figs. 4 and 5 and Supplementary Figs. 3 and 5-9 are provided as Supplementary Software 1 and Supplementary Software 2 .

Received: 13 March 2019; Accepted: 9 October 2019; Published online: 18 November 2019

\section{References}

1. Daszak, P., Cunningham, A. A. \& Hyatt, A. D. Emerging infectious diseases of wildlife-threats to biodiversity and human health. Science 287, 443-449 (2000).

2. Brochier, B. et al. Large-scale eradication of rabies using recombinant vaccinia-rabies vaccine. Nature 354, 520-522 (1991).

3. Slate, D. et al. Oral rabies vaccination in North America: opportunities, complexities, and challenges. PLoS Negl. Trop. Dis. 3, e549 (2009).

4. Hoffmann, A. et al. Successful establishment of Wolbachia in Aedes populations to suppress dengue transmission. Nature 476, 454-457 (2011).

5. Sinkins, S. P. \& Gould, F. Gene drive systems for insect disease vectors. Nat. Rev. Genet. 7, 427-435 (2006).

6. Aliota, M. T., Peinado, S. A., Velez, I. D. \& Osorio, J. E. The $w$ Mel strain of Wolbachia reduces transmission of Zika virus by Aedes aegypti. Sci. Rep. 6, 28792 (2016).

7. Basinski, A. J. et al. Evaluating the promise of recombinant transmissible vaccines. Vaccine 36, 675-682 (2018).

8. Bull, J. J., Smithson, M. W. \& Nuismer, S. L. Transmissible viral vaccines. Trends Microbiol. 26, 6-15 (2018).

9. Minor, P. Vaccine-derived poliovirus (VDPV): impact on poliomyelitis eradication. Vaccine 27, 2649-2652 (2009).

10. Behdenna, A. et al. Transmission ecology of canine parvovirus in a multi-host, multi-pathogen system. Proc. R. Soc. B Biol. Sci. 286, 20182772 (2019).

11. Nuismer, S. L. et al. Eradicating infectious disease using weakly transmissible vaccines. Proc. R. Soc. Lond. B Biol. Sci. 283, 20161903 (2016).
12. Torres, J. M. et al. First field trial of a transmissible recombinant vaccine against myxomatosis and rabbit hemorrhagic disease. Vaccine 19, 4536-4543 (2001).

13. Schneider, M. C. et al. Potential force of infection of human rabies transmitted by vampire bats in the Amazonian region of Brazil. Am. J. Trop. Med. Hyg. 55, 680-684 (1996).

14. Belotto, A., Leanes, L. F., Schneider, M. C., Tamayp, H. \& Correa, E. Overview of rabies in the Americas. Virus Res. 111, 5-12 (2005)

15. Benavides, J. A., Paniagua, E. R., Hampson, K., Valderrama, W. \& Streicker, D. G. Quantifying the burden of vampire bat rabies in Peruvian livestock. PLoS Negl. Trop. Dis. 11, e0006105 (2017).

16. Schneider, M. C. et al. Rabies transmitted by vampire bats to humans: an emerging zoonotic disease in Latin America? Rev. Panam. Salud Públ. 25, 260-269 (2009).

17. Linhart, S. B., FloresCrespo, R. \& Mitchell, G. C. Control of vampire bats by topical application of an anticoagulant, chlorophacinone. Bol. OSP 6, 31-38 (1972).

18. Fornes, A. et al. Control of bovine rabies through vampire bat control. J. Wildl. Dis. 10, 310-316 (1974).

19. Streicker, D. G. et al. Ecological and anthropogenic drivers of rabies exposure in vampire bats: implications for transmission and control. Proc. R. Soc. B Biol. Sci. 279, 3384-3392 (2012).

20. Blackwood, J. C., Streicker, D. G., Altizer, S. \& Rohani, P. Resolving the roles of immunity, pathogenesis, and immigration for rabies persistence in vampire bats. Proc. Natl Acad. Sci. USA 110, 20837-20842 (2013).

21. Donnelly, C. A. et al. Impact of localized badger culling on tuberculosis incidence in British cattle. Nature 426, 834-837 (2003).

22. Aguilar-Setién, A. et al. Experimental rabies infection and oral vaccination in vampire bats (Desmodus rotundus). Vaccine 16, 1122-1126 (1998).

23. Aguilar-Setién, A. et al. Vaccination of vampire bats using recombinant vaccinia-rabies virus. J. Wildl. Dis. 38, 539-544 (2002).

24. Stading, B. et al. Protection of bats (Eptesicus fuscus) against rabies following topical or oronasal exposure to a recombinant raccoon poxvirus vaccine. PLoS Negl. Trop. Dis. 11, e0005958 (2017).

25. Almeida, M., Martorelli, L., Aires, C., Barros, R. \& Massad, E. Vaccinating the vampire bat Desmodus rotundus against rabies. Virus Res. 137, 275-277 (2008)

26. Cagnacci, F., Massei, G., Coats, J., DeLeeuw, A. \& Cowan, D. P. Long-lasting systemic bait markers for Eurasian badgers. J. Wildl. Dis. 42, 892-896 (2006).

27. Fry, T. L., Atwood, T. \& Dunbar, M. R. Evaluation of rhodamine B as a biomarker for raccoons. Hum. Wildl. Interact. 4, 275-282 (2010).

28. Fernandez, J. R. R. \& Rocke, T. E. Use of rhodamine B as a biomarker for oral plague vaccination of prairie dogs. J. Wildl. Dis. 47, 765-768 (2011).

29. Clay, C. A., Lehmer, E. M., Previtali, A., St Jeor, S. \& Dearing, M. D. Contact heterogeneity in deer mice: implications for Sin Nombre virus transmission. Proc. R. Soc. B Biol. Sci. 276, 1305-1312 (2009).

30. Hoyt, J. R. et al. Cryptic connections illuminate pathogen transmission within community networks. Nature 563, 710-713 (2018).

31. Hampson, K. et al. Transmission dynamics and prospects for the elimination of canine rabies. PLoS Biol. 7, e1000053 (2009).

32. Amengual, B., Bourhy, H., López-Roíg, M. \& Serra-Cobo, J. Temporal dynamics of European bat lyssavirus type 1 and survival of Myotis myotis bats in natural colonies. PLOS ONE 2, 0000566 (2007)

33. Streicker, D. G. et al. Host-pathogen evolutionary signatures reveal dynamics and future invasions of vampire bat rabies. Proc. Natl Acad. Sci. USA 113, 10926-10931 (2016).

34. Benavides, J. A., Valderrama, W. \& Streicker, D. G. Spatial expansions and travelling waves of rabies in vampire bats. Proc. R. Soc. Lond. B Biol. Sci. 283, 20160328 (2016)

35. Choisy, M. \& Rohani, P. Harvesting can increase severity of wildlife disease epidemics. Proc. R. Soc. B Biol. Sci. 273, 2025-2034 (2006).

36. Gomes, M. N., Uieda, W. \& Latorre, M. Influence of sex differences in the same colony for chemical control of vampire Desmodus rotundus (Phyllostomidae) populations in the state of Sao Paulo, Brazil. Pesqui. Vet. Bras. 26, 38-43 (2006)

37. Wilkinson, G. S. Social grooming in the common vampire bat, Desmodus rotundus. Anim. Behav. 34, 1880-1889 (1986).

38. Carter, G. \& Leffer, L. Social grooming in bats: are vampire bats exceptional? PLOS ONE 10, e0138430 (2015).

39. Bergner, L. M. et al. Using noninvasive metagenomics to characterize viral communities from wildlife. Mol. Ecol. Res. 19, 128-143 (2019).

40. Thompson, R. D., Elias, D. J. \& Mitchell, G. C. Effects of vampire bat control on bovine milk production. J. Wildl. Manage. 41, 736-739 (1977).

41. Hardy, C. et al. Biological control of vertebrate pests using virally vectored immunocontraception. J. Reprod. Immunol. 71, 102-111 (2006).

42. Beyer, H. L. et al. The implications of metapopulation dynamics on the design of vaccination campaigns. Vaccine 30, 1014-1022 (2012).

43. Stading, B. R. et al. Infectivity of attenuated poxvirus vaccine vectors and immunogenicity of a raccoonpox vectored rabies vaccine in the Brazilian free-tailed bat (Tadarida brasiliensis). Vaccine 34, 5352-5358 (2016). 
44. Langwig, K. E. et al. Resistance in persisting bat populations after white-nose syndrome invasion. Phil. Trans. R. Soc. B Biol. Sci. 372, 20160044 (2017).

45. Plowright, R. K. et al. Urban habituation, ecological connectivity and epidemic dampening: the emergence of Hendra virus from flying foxes (Pteropus spp.). Proc. R. Soc. B Biol. Sci. 278, 3703-3712 (2011).

46. Hayman, D. T. Biannual birth pulses allow filoviruses to persist in bat populations. Proc. R. Soc. B Biol. Sci. 282, 20142591 (2015).

47. Rocke, T. E. et al. Virally-vectored vaccine candidates against white-nose syndrome induce anti-fungal immune response in little brown bats (Myotis lucifugus). Sci. Rep. 9, 6788 (2019).

48. Marzi, A. et al. Cytomegalovirus-based vaccine expressing Ebola virus glycoprotein protects nonhuman primates from Ebola virus infection. Sci. Rep. 6, 21674 (2016).

49. Pallister, J. et al. A recombinant Hendra virus G glycoprotein-based subunit vaccine protects ferrets from lethal Hendra virus challenge. Vaccine 29, 5623-5630 (2011).

50. Olival, K. \& Hayman, D. Filoviruses in bats: current knowledge and future directions. Viruses 6, 1759-1788 (2014).

51. Schnabel, Z. E. The estimation of total fish population of a lake. Am. Math. Monthly 45, 348-352 (1938).

52. Anthony, E. L. P. in Ecological and Behavioral Methods for the Study of Bats (ed. Kunz, T. H.) 47-58 (Smithsonian Institution Press, 1988).

53. Constantine, D. G., Tierkel, E. S., Kleckner, M. D. \& Hawkins, D. M. Rabies in New Mexico cavern bats. Public Health Rep. 83, 303-316 (1968).

54. Delpietro, H., Russo, R., Carter, G., Lord, R. \& Delpietro, G. Reproductive seasonality, sex ratio and philopatry in Argentina's common vampire bats. R. Soc. Open Sci. 4, 160959 (2017).

55. Stading, B. Development of a Novel Rabies Mosaic Antigen and the Use of Attenuated Poxviruses as Vaccine Vectors in Bats (University of WisconsinMadison, 2015).

\section{Acknowledgements}

K.M.B. was supported by NIH award F32AI134016, and computational resources were provided by NIH award U01GM110712. D.G.S. was supported by a Sir Henry Dale Fellowship that was jointly funded by the Wellcome Trust and Royal Society (102507/Z/13/Z). Additional funding was provided via a Challenge Grant from the Royal Society to D.G.S., T.E.R., J.E.O., C.S. and N.F. (CH160097). The authors are grateful to D. Walsh, M. Viana and the Streicker Group for comments on earlier versions of this manuscript. Any use of trade, product or firm names does not imply endorsement by the US Government.

\section{Author contributions}

D.G.S., T.E.R. and J.E.O. conceived and designed the experiments. R.C.A., C.T. and J.E.C. performed the experiments. K.M.B. and D.G.S. analysed the data. T.E.R., J.E.O., W.V., C.S. and N.F. contributed materials and/or analysis tools. K.M.B. and D.G.S. wrote the first draft of the paper. All authors contributed revisions.

\section{Competing interests}

The authors declare no competing interests.

\section{Additional information}

Supplementary information is available for this paper at https://doi.org/10.1038/ s41559-019-1032-X.

Correspondence and requests for materials should be addressed to K.M.B. or D.G.S. Reprints and permissions information is available at www.nature.com/reprints. Publisher's note Springer Nature remains neutral with regard to jurisdictional claims in published maps and institutional affiliations.

(C) The Author(s), under exclusive licence to Springer Nature Limited 2019 


\section{natureresearch}

\section{Reporting Summary}

Nature Research wishes to improve the reproducibility of the work that we publish. This form provides structure for consistency and transparency in reporting. For further information on Nature Research policies, see Authors \& Referees and the Editorial Policy Checklist.

\section{Statistics}

For all statistical analyses, confirm that the following items are present in the figure legend, table legend, main text, or Methods section.

$\mathrm{n} / \mathrm{a} \mid$ Confirmed

$\square$ \The exact sample size $(n)$ for each experimental group/condition, given as a discrete number and unit of measurement

$\square \bigotimes$ A statement on whether measurements were taken from distinct samples or whether the same sample was measured repeatedly

The statistical test(s) used AND whether they are one- or two-sided

$\bigotimes$ Only common tests should be described solely by name; describe more complex techniques in the Methods section.

$\square$ \ A description of all covariates tested

$\square$ \ A description of any assumptions or corrections, such as tests of normality and adjustment for multiple comparisons

$\square$ A full description of the statistical parameters including central tendency (e.g. means) or other basic estimates (e.g. regression coefficient) AND variation (e.g. standard deviation) or associated estimates of uncertainty (e.g. confidence intervals)

$\square$ For null hypothesis testing, the test statistic (e.g. $F, t, r$ ) with confidence intervals, effect sizes, degrees of freedom and $P$ value noted

$\square$ Give P values as exact values whenever suitable.

Х $\square$ For Bayesian analysis, information on the choice of priors and Markov chain Monte Carlo settings

Х $\square$ For hierarchical and complex designs, identification of the appropriate level for tests and full reporting of outcomes

$\bigotimes \square$ Estimates of effect sizes (e.g. Cohen's $d$, Pearson's $r$ ), indicating how they were calculated

Our web collection on statistics for biologists contains articles on many of the points above.

\section{Software and code}

Policy information about availability of computer code

Data collection

No software was used to collect the data

Data analysis

All code was run on R version 3.4.4 (2018-03-15) -- "Someone to Lean On", with package 'deSolve', version 1.20. Package 'Matrix' is version 1.2-12. The R scripts used to estimate RB transfer rates (Supplementary Figure 3, Supplementary Table 2) and to carry out the epidemiological modeling (Figures $4 \& 5$ and Supplementary Figures 5-9) are provided as Supplementary Information.

For manuscripts utilizing custom algorithms or software that are central to the research but not yet described in published literature, software must be made available to editors/reviewers. We strongly encourage code deposition in a community repository (e.g. GitHub). See the Nature Research guidelines for submitting code \& software for further information.

\section{Data}

Policy information about availability of data

All manuscripts must include a data availability statement. This statement should provide the following information, where applicable:

- Accession codes, unique identifiers, or web links for publicly available datasets

- A list of figures that have associated raw data

- A description of any restrictions on data availability

The UV transfer and RB transfer data are available on Dryad (doi: 10.5061/dryad.64t161m). These data were used to generate Figures 2 \& 3 and Supplementary Figures 1 \& 2. Additional files have also been provided with the UV transfer and RB transfer separately (Data.Figure1_RB.xls and Data.Figure2_UV.xls). 


\section{Field-specific reporting}

Please select the one below that is the best fit for your research. If you are not sure, read the appropriate sections before making your selection.
Life sciences
Behavioural \& social sciences
Ecological, evolutionary \& environmental sciences

For a reference copy of the document with all sections, see nature.com/documents/nr-reporting-summary-flat.pdf

\section{Ecological, evolutionary \& environmental sciences study design}

All studies must disclose on these points even when the disclosure is negative.

Study description

Field studies were carried out in wild vampire bat colonies in Peru to monitor the spread of two topically-applied biomarkers: a UV powder, which was intended to trace contacts among demographic groups and a rhodamine b gel that was intended to simulate the transfer and ingestion of self-spreading disease control agents. The studies were carried out in three replicate bat colonies, which ranged in size from 207 to 257 individuals (according to mark-recapture analysis). Data were used to estimate parameters that were included in mathematical models.

Research sample

The research sample consisted of common vampire bats (Desmodus rotundus) captured at 3 sites near Lima, Peru. Individuals were male and female bats of adult, subadult, and juvenile age. This species is the most appropriate for the research question posed which involved the dynamics of rabies transmission and control in vampire bats. The colonies we studied are of typical size, age and sex distributions for this species in Peru. The research sample is therefore designed to apply more broadly to other populations of this species. Existing data from previous field studies and experiments in captive vampire bat were used to assign parameters in mathematical modeling. The parameter values used and their associated literature references are provided in Supplementary Table 3.

Sampling strategy

Sample sizes for field studies depended on capture success of vampire bats, which was largely beyond our control. However, we selected highly accessible bat colonies where capture successes would be maximized and standardized capture efforts across sites. Our decision to carry out our field experiment in three replicate colonies was based on the logistical constraints of field work, since this was the maximum number of colonies that could be monitored simultaneously by a single field team working full time.

Data collection

Field data were collected by predominately co-authors Carlos Tello and Jorge Carrera with some early assistance from other coauthors (Bakker, Streicker, Osorio, Rocke). The rhodamine study was carried out once per bat colony since rhodamine is a long lasting marker, so transfers from repeat treatments would not be distinguishable. Rhodamine transfers were monitored by catching bats for one month after application across 4-5 sampling instances. Uptake of rhodamine was assessed by plucking hair samples from captured bats and microscopy (see below). The UV contact tracing was carried out 3 times per bat colony since powders are only detectable for several days after application. UV transfers were monitored for 2 nights after each application by inspecting bats with UV lights prior to handling (to avoid any potential contamination from gloves). Supplementary Table 1 contains a timeline of all field activities.

Laboratory analysis of hair samples was carried out by co-authors Carlos Tello and Rachel Abbott.

Timing and spatial scale Data were collected between January 31, 2017 and July 30, 2017 in three bat colonies in the Department of Lima, Peru. Sites were separated by 54 to $200 \mathrm{~km}$, a distance that is well beyond the expected dispersal ability of vampire bats, making our colonies independent. The LMA5 colony was studied between April 20, 2017 and July 27, 2017. The LMA6 colony was studied between April 22, 2017 and July 24, 2017. The LMA12 colony was studied between January 31, 2017 and July 30, 2017.

Data exclusions No data were excluded.

Reproducibility

Each of our bat colonies is considered a replicate of the rhodamine $b$ transfer estimate. We faced logistical challenges in colony LMA12 since a large fraction of that colony abandoned the roost site. Nevertheless we include these data in all analyses for completeness. The UV studies were repeated 3 times per bat colony ( 9 total).

Randomization

We aimed to infer rates that would be most relevant to real-world campaigns, thus randomization of biomarker application was not appropriate. Instead we apply rhodamine or UV powders to all captured individuals on designated capture days/nights.

Blinding

Individuals conducing the microscopy were blinded to the whether hair samples came from rhohdamine treated bats. Individuals carrying out the UV detection were blinded to whether UV had been applied to those bats.

Did the study involve field work? \Yes $\square$ No

\section{Field work, collection and transport}

Field conditions

Location
The study was carried out in the Department of Lima, Peru which is largely a subtropical coastal desert with little appreciable rainfall. Average minimum/maximum temperatures between January and July are 16-21 C and 19-27 C.

Field studies were carried out in three vampire bat roosts in the Barranca (LMA5, $-10.6415,-77.8160$; elevation $=65.6$ meters), Huaura (LMA6, $-11.0555,-77.4594$, elevation=354.7), and Lima (LMA12, $-12.1833,-76.8500$, elevation=202 meters) districts. 


\section{Reporting for specific materials, systems and methods}

We require information from authors about some types of materials, experimental systems and methods used in many studies. Here, indicate whether each material, system or method listed is relevant to your study. If you are not sure if a list item applies to your research, read the appropriate section before selecting a response.

Materials \& experimental systems

\begin{tabular}{l|l}
\hline n/a & Involved in the study \\
$\square$ & $\square$ Antibodies \\
$\square$ & $\square$ Eukaryotic cell lines \\
$\square$ & $\square$ Palaeontology \\
$\square$ & $\square$ Animals and other organisms \\
$\square$ & $\square$ Human research participants \\
$\square$ & $\square$ Clinical data
\end{tabular}

\begin{tabular}{l|l}
\multicolumn{2}{l}{ Methods } \\
\hline n/a & Involved in the study \\
$\square$ & $\square$ ChIP-seq \\
$\bigotimes$ & $\square$ Flow cytometry \\
$\square$ & $\square$ MRI-based neuroimaging
\end{tabular}

\section{Animals and other organisms}

Policy information about studies involving animals; ARRIVE guidelines recommended for reporting animal research

Laboratory animals

Wild animals

Field-collected samples

Ethics oversight
No laboratory animals were used in this study.

Wild common vampire bats (Desmodus rotundus) were captured in Peru. Both males and females of adult, subadult and juvenile age were sampled. Bats were captured using hand nets within roosts during the day and using mist nets to capture bats exiting to forage at night. Nocturnal captures lasted from approximately 18:00 - 6:00, and nets were checked every 30 minutes. Animals were held in individual bags prior to processing, and were released in the same area where they were captured immediately after sampling and/or application of biomarkers.

No laboratory work was performed with field collected animals. Animals were released at the site of capture after sampling. Hair samples were collected from bats and analyzed by microscopy.

This research was performed under approval of University of Glasgow School of Veterinary Medicine Animal Ethics Committee, approval number, 25A/18.

Note that full information on the approval of the study protocol must also be provided in the manuscript. 